\title{
Industrie et environnement en Allemagne
}

Manufacturing industry and environment in Germany

Industrie und Umwelt in Deutschland

\section{Christian Schulz}

\section{(2) OpenEdition}

\section{Journals}

Édition électronique

URL : http://journals.openedition.org/rge/839

ISSN : 2108-6478

\section{Éditeur}

Association des géographes de l'Est

Édition imprimée

Date de publication : 1 janvier 2007

ISSN : 0035-3213

\section{Référence électronique}

Christian Schulz, «Industrie et environnement en Allemagne », Revue Géographique de l'Est [En ligne], vol. 47 / 1 | 2007, mis en ligne le 22 décembre 2009, consulté le 07 septembre 2020. URL : http:// journals.openedition.org/rge/839

Ce document a été généré automatiquement le 7 septembre 2020

Tous droits réservés 


\title{
Industrie et environnement en
} Allemagne

\author{
Manufacturing industry and environment in Germany \\ Industrie und Umwelt in Deutschland
}

Christian Schulz

L'auteur tient à remercier Michel Deshaies d'avoir bien voulu prendre en charge la traduction du manuscrit allemand.

\section{Introduction}

1 En Allemagne, comme dans beaucoup d'autres pays industrialisés, une prise de conscience concernant la protection de l'environnement dans l'industrie s'est développée depuis une trentaine d'années. Dans beaucoup de branches, on s'est rendu compte que les investissements destinés à la modernisation environnementale pouvaient être compatibles avec les objectifs économiques des entreprises, que ce soit par des effets directs d'économie (par exemple de la consommation d'énergie, des ressources et des coûts d'épuration), ou bien par des effets induits comme ceux générés par les avantages que procurent le fait d'être précurseur sur un marché. Cependant, ce changement ne repose qu'en partie sur l'engagement volontaire des entreprises, mais doit plutôt être considéré comme le résultat d'un processus de négociation politique et sociale dont les mécanismes divers ont conduit à la situation actuelle.

2 L'objectif de cet article est d'abord de donner un aperçu de l'importance accordée à l'environnement dans la production industrielle en Allemagne. On montrera aussi les stratégies d'action de la protection de l'environnement industriel ainsi que l'importance économique croissante du secteur de l'environnement. Enfin, on terminera par un bilan critique. 


\section{Les problèmes environnementaux liés à la production industrielle}

3 Avec la réunification allemande qui a provoqué la fermeture de nombreuses entreprises industrielles et la tendance à la désindustrialisation résultant de l'évolution économique générale, il y a eu sans aucun doute une diminution de l'importance de la production industrielle qui a eu des effets bénéfiques sur la situation de l'environnement. Cependant, le secteur manufacturier en Allemagne joue non seulement un rôle déterminant dans la production économique ( $21 \%$ de la population active; $30 \%$ du PIB en 2005, DESTATIS 2006a), mais il est aussi avec le secteur énergétique et celui des transports une des plus importantes sources d'émissions de polluants dans l'atmosphère (UBA, 2006b). Par ses rejets, l'industrie contribue aussi (avec les effluents urbains) beaucoup à la pollution chimique des nappes phréatiques et des eaux de surface (DESTATIS 2005a). A cela s'ajoute le réchauffement par les rejets d'eaux de refroidissement.

4 Il en est de même en ce qui concerne la consommation d'espace. En 2001, celle-ci représentait $4460 \mathrm{~km} 2$ pour l'industrie manufacturière et le secteur de la production et de la distribution d'énergie et $24799 \mathrm{~km} 2$ pour l'habitat (DESTATIS 2005b). Avec 7900 Petajoules pour la consommation finale d'énergie, le secteur manufacturier est aussi nettement devant les foyers (3900 PJ) et le secteur des transports (environ $2800 \mathrm{PJ}$, DESTATIS 2003 et 2005c).

5 Malgré la part importante de l'industrie dans les problèmes environnementaux actuels, la pollution a sensiblement diminué au cours des dernières décennies. Les raisons de cette évolution positive vont être maintenant présentées.

\section{Les stratégies de remédiation}

Dans les années 1970 l'Allemagne a mis en place sa propre politique de protection de l'environnement, qui est en particulier consacrée à la protection de l'environnement industriel. Une succession de dispositions juridiques, comme la loi fédérale sur la protection contre les émissions polluantes (Bundesimmissionsschutzgesetz) de 1974, l'ordonnance sur les grandes installations thermiques (Großfeuerungsanlagenverordnung) de 1983, ou encore la loi sur l'air (TA Luft) de 19861 ont provoqué des mesures d'adaptation dans le secteur manufacturier et ont contribué à une amélioration sensible de l'environnement. En dehors de la dépollution de l'air, on a beaucoup investi dans les domaines de l'épuration des eaux et dans le traitement des déchets (tableau 1).

7 Il n'est pas étonnant que les branches industrielles qui ressortent soient précisément celles qui, en raison de leur procédé de production (par exemple la sidérurgie), ou des matières premières employées (la pétrochimie), sont à l'origine de fortes pollutions locales et ont été en conséquence la cible des critiques des habitants, des associations de protection de l'environnement et des médias.

Tableau 1. Coûts annuels liés à la protection de l'environnement en 2004 [1000 euros]

\begin{tabular}{|l|l|l|l|l|l|}
\hline Filière & Déchets & Eaux & Air & Autres & Total \\
\hline
\end{tabular}




\begin{tabular}{|l|l|l|l|l|l|}
\hline Industrie alimentaire & 242891 & 476473 & 47661 & 12830 & 779855 \\
\hline Pâtes et papiers & 173166 & 196539 & 44023 & 4336 & 418064 \\
\hline Imprimeries & 45104 & 16006 & 27717 & 1929 & 90756 \\
\hline Cokeries, raffineries & 60539 & 324890 & 645049 & 38853 & 1069331 \\
\hline Produits chimiques & 663827 & 858045 & 404420 & 71296 & 1997588 \\
\hline Caoutchouc, plastiques & 121708 & 42724 & 43607 & 5887 & 213926 \\
\hline Verres et céramiques & 105583 & 52599 & 113942 & 17867 & 289991 \\
\hline Métallurgie & 299213 & 399961 & 532478 & 42001 & 1273653 \\
\hline Construction mécanique & 141872 & 100284 & 40059 & 16601 & 298816 \\
\hline Automobiles & 352747 & 327888 & 176900 & 65218 & 922753 \\
\hline Autres & 421152 & 321158 & 115795 & 35562 & 893667 \\
\hline Total & 2627802 & 3116567 & 2191651 & 312380 & 8248400 \\
\hline
\end{tabular}

8 En dehors des dispositions juridiques et des instruments de contrôle et de taxation sans lesquels il $n^{t} y$ aurait probablement jamais eu de changement de stratégie dans l'industrie, à partir des années 1990 l'Etat a mis en place de plus en plus d'instruments d'incitation et l'industrie a spontanément pris des engagements. Les premiers ont accordé un soutien financier et/ou administratif (par des baisses d'impôts, des aides à l'investissement, des dispositifs d'autorisation simplifiés) aux entreprises industrielles qui remplissaient les obligations juridiques, ou qui s'engageaient dans de nouvelles voies pour la protection de l'environnement (par exemple l'emploi de technologies innovantes). Cela va souvent de pair avec des engagements individuels ou de branches, comme l'a fait très tôt l'industrie chimique dans le cadre de son programme international de responsabilité dans lequel les entreprises participantes s'obligent à respecter un code de bonnes pratiques (Moffet, Bregha, 1999). Les entreprises sont d'autant plus disposées à remplir de tels engagements qu'en dehors des effets sur leur image et des facilités administratives signalées, elles peuvent en obtenir l'avantage d'être précurseur sur un marché. C'est le cas par exemple quand une entreprise est la seule à proposer un produit bon pour l'environnement (par exemple le filtre à particules pour les moteurs diesel), ou quand elle est la première à utiliser un procédé de fabrication particulièrement bon pour l'environnement (par exemple la fabrication de papier sans chlore) et qu'elle profite alors d'avantages de compétitivité par rapport à ses concurrents « en retard». 
Figure 1 : Participation à l'EMAS et à ISO 14001 dans les pays membres de l'UE en juillet 2006 (graphique : C. Schulz ; sources : EC, 2006, UBA, 2006c).

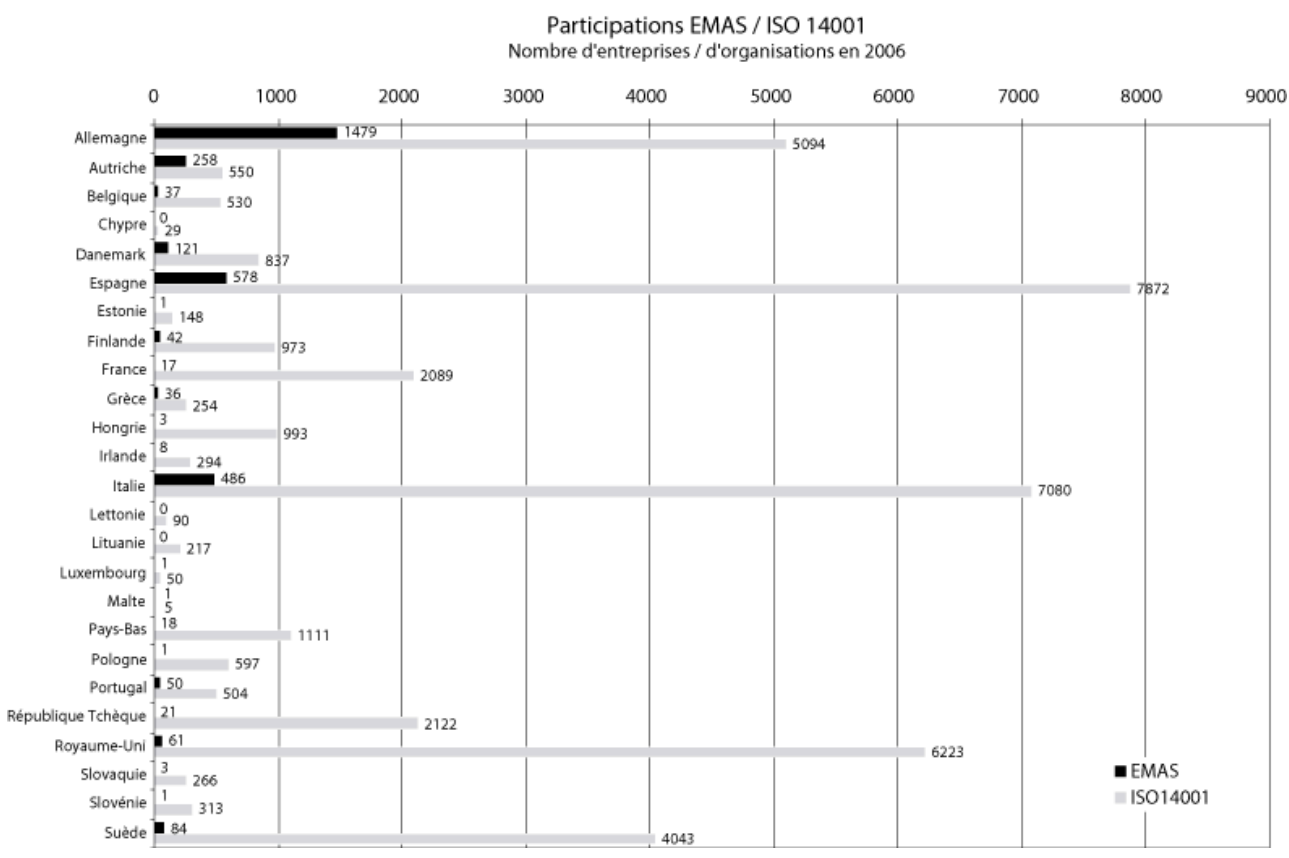

Parmi les engagements librement consentis, il faut compter la mise en place de systèmes de management environnemental et la participation à des évaluations indépendantes des activités dans l'environnement (audits) comme la norme internationale ISO 14001 ou le système européen EMAS2 (éco-audit). Les deux systèmes ont remporté un grand succès dans l'industrie allemande. Alors que dans les années 1990 de nombreuses entreprises ont d'abord adopté les certificats EMAS, c'est la norme internationale ISO 14001 qui est devenue récemment la plus importante (et intégré dans le système EMAS, après sa révision). Actuellement, plus de 5000 entreprises allemandes sont certifiées ISO 14001. 
Figure 2 : Répartition des firmes certifiées EMAS / Eco-Audit sur les Länder en janvier 2006 (cartographie : C. Schulz; source : Umweltgutachterausschuss, 2006a)

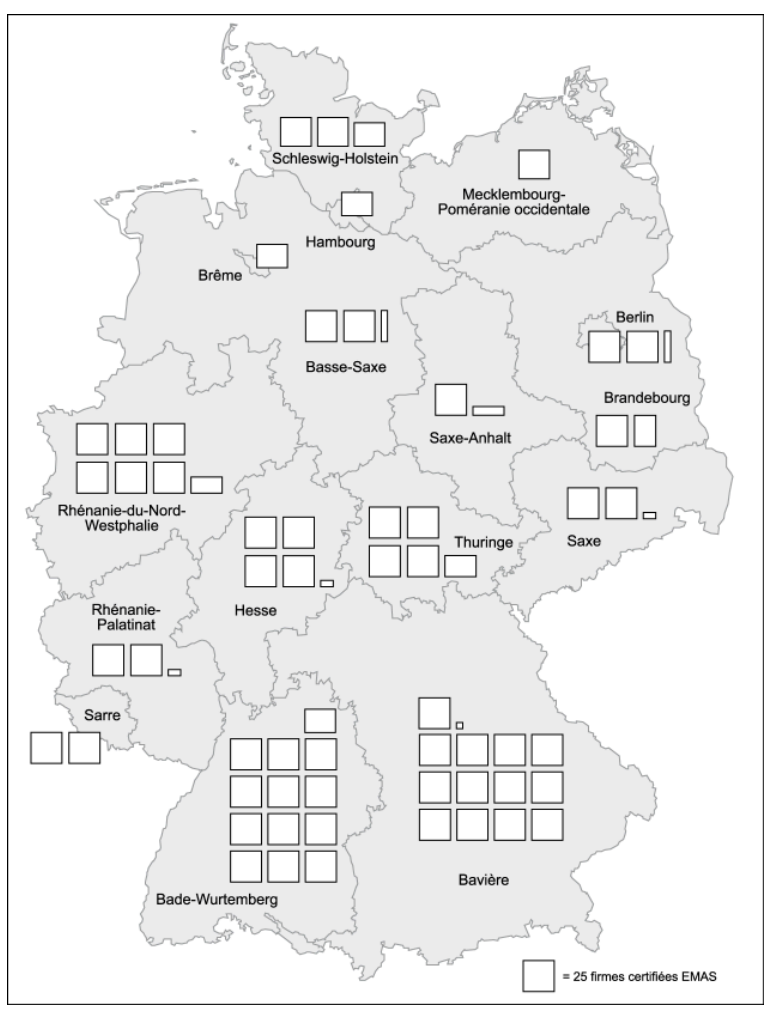

10 En dehors des mesures de protection, ce sont surtout les changements des conditions du secteur énergétique qui ont influencé fortement la situation de l'environnement et ont conduit en même temps au développement de nouveaux segments sur le marché de la protection de l'environnement. La loi sur les énergies renouvelables (ErneuerbareEnergien-Gesetz, EEG) promulguée en 2000 par le gouvernement de la coalition rougeverte et les prix garantis qu'elle prévoit ont entraîné un développement rapide de l'utilisation du vent, du soleil et de la biomasse pour la production d'énergie. En 2006, la part des énergies renouvelables dans la consommation totale en énergie s'élevait à $7,4 \%$ (par rapport à $6,6 \%$ en 2005), la part dans la consommation brute en électricité à 11,8 \% (par rapport à 10,4 en 2005 ; BMU 2007). La loi allemande EEG a ainsi servi de modèle pour des lois comparables dans d'autres États industrialisés (par exemple l'Espagne), mais aussi dans des États en voie de développement (par exemple la Chine).

\section{Technologies et services environnementaux : des marchés prometteurs}

Au début de l'année 2006, il y avait environ 1,5 million de personnes qui travaillaient dans le secteur de la protection de l'environnement en Allemagne (contre 200000 en 1975). Cela représente presque $4 \%$ de la population active, plus qu'actuellement dans la construction automobile (BUND, 2006A). La branche de la protection de l'environnement est ainsi devenue un facteur important et de mieux en mieux reconnu du marché du travail. La branche profite ainsi de l'intérêt croissant des clients étrangers pour les techniques de l'environnement développées et fabriquées en Allemagne, ainsi que des services spécialisés. Les techniques de l'environnement (47 
000 emplois), mais aussi de plus en plus le secteur des services environnementaux de haut niveau (Schulz 2005a, 2005b) sont devenus un important produit d'exportation et font que, sur ce marché, l'Allemagne fait partie des plus grands exportateurs du monde.

En dehors des pays européens, les exportations se font surtout vers les pays en voie de développement d'Asie et d'Amérique latine. La Chine notamment est devenue un important marché en raison de la dynamique de son industrialisation et des problèmes environnementaux croissants (Heymann 2006).

13 Parmi les produits les plus connus dans ce domaine, il y a bien sûr les éoliennes fabriquées en Allemagne, par exemple par les firmes ENERCON, Fuhrländer, Nordex, REpower Systems ou Siemens Wind Power. Avec un chiffre d'affaires de 4 Mrd. _ et 70 000 emplois (en comptant la sous-traitance) la branche a atteint un record en 2006 et domine le marché mondial. La dynamique de cette branche économique dans les années quatre-vingt-dix est à corréler avec la croissance de l'installation des éoliennes en Allemagne (fin 2006: 18685 éoliennes pour 20622 MW, dont 2233 MW installés en 2006). Depuis lors, le secteur profite de plus en plus des exportations sur les marchés en croissance, en particulier aux Etats-Unis ( 2800 MW installés en 2006), en Inde (1 500 $\mathrm{MW})$ et en Chine ( $1000 \mathrm{MW})$. Au total, le marché mondial a augmenté de $30 \%$ en 2006 et donc plus vite que le marché intérieur (BWE 2007). Depuis quelques années, l'industrie allemande des éoliennes réalise la majeure partie de son chiffre d'affaires à l'étranger (plus de $60 \%$ en 2004 avec une tendance croissante, BWE/VDMA 2007).

14 L'évolution est également impressionnante dans une autre branche énergétique, celle de l'énergie solaire. La Conergy AG de Hambourg ainsi que la Solarwold AG de Bonn comptent parmi les plus gros fournisseurs de systèmes de transformation de l'énergie solaire en Europe et ont fortement accru leur importance internationale par des acquisitions en Europe et en Amérique du Nord. La croissance du marché de cette branche en Allemagne a atteint $20 \%$ en 2006. En comptant les sous-traitants, il y a environ 5000 entreprises employant 42500 personnes dans ce secteur d'activité. En 2005 ils ont réalisé un chiffre d'affaires de 3,7 Mrd. _, dont $80 \%$ dans le domaine des cellules photovoltaïques (Intersolar 2006). Dans ces conditions, il n'est donc pas étonnant que la plus grande foire spécialisée, Intersolar, se déroule chaque année en Allemagne (à Fribourg en Brisgau jusqu'en 2007 et à partir de 2008 à Munich pour des problèmes de place) et en 2007 on attend plus de 500 exposants.

15 Parmi les autres piliers et articles d'exportation du secteur de l'environnement en Allemagne, il y a le domaine plutôt classique des équipements pour l'épuration de l'eau, la filtration de l'air, la protection contre le bruit, etc. Depuis quelques années, le secteur des services spécialisés en environnement est aussi en forte croissance pour l'exportation. On peut citer en exemple une entreprise de services très exportatrice, la TÜV Rheinland, qui a acquis une réputation internationale en proposant des prestations sous forme de conseils dans le domaine du management de l'environnement. Elle entretient un réseau de filiales en Chine (TÜV Rheinland Group China) où elle a été la première entreprise à obtenir la certification ISO 14001 suivant le standard de l'agence nationale d'accréditation CACEB (China Accreditation Committee for Environmental Management System Certification Bodies) (TÜV 2005). 


\section{Conclusions} tes nouvelles directives européennes. L'exemple de la très contestée directive Natura 2000 témoigne non seulement des conflits de compétence entre et à l'intérieur des Länder, mais elle a été aussi fortement entravée par l'intervention de représentants économiques. Cela a été encore plus net dans le cas de la directive REACH3 qui, avant même son adoption, a été fortement critiquée et combattue par les syndicats et les lobbies des branches concernées.

Il est encore difficile d'évaluer dans quelle mesure le changement récent de gouvernement de la coalition rouge-verte à la grande coalition de la CDU et du SPD va influencer la politique de l'environnement concernant l'industrie. On peut s'attendre en particulier à des changements concernant la politique énergétique, puisque la CDU/ CSU remet en question la sortie du nucléaire décidée par le gouvernement précédent. Mais dans le même temps, le gouvernement fédéral a désigné justement le problème énergétique comme étant le thème principal de sa présidence du conseil européen au cours de la première moitié de l'année 2007 et il a annoncé qu'il allait accorder une attention particulière au développement des énergies renouvelables. On a récemment critiqué la mise en application du deuxième plan national d'allocation (NAP) de droits d'émissions de $\mathrm{CO} 2$ qui (tout comme lors de sa première édition en 2004) prévoit une attribution totalement gratuite à des entreprises industrielles et à des producteurs d'énergie, alors que la directive européenne sur les échanges de droits d'émission accorde une mise aux enchères de $10 \%$ de ces droits pour la deuxième période de 2008 à 2012. L'accroissement d'une partie des droits d'émission rendrait beaucoup moins attractif pour les producteurs d'électricité les gains provenant du développement des énergies renouvelables (windfall profit) (BUND 2006b). Au total, les réglementations de NAP II sont plus transparentes et leurs effets sont plus faciles à estimer que ce n'était le cas avec NAP I où il y avait beaucoup de réglementations particulières. Un point particulièrement critique est que, comme auparavant, la répartition des droits d'émission pour les nouvelles implantations est toujours modulée en fonction de la source d'énergie. Ainsi, les centrales thermiques au lignite et au charbon se voient accorder deux fois plus de droits d'émission de $\mathrm{CO} 2$ par kWh que les centrales à gaz modernes, avec du coup deux fois plus de nuisances pour le climat. Par là même, on favorise les investissements dans de nouvelles centrales thermiques au charbon qui, pendant des années, vont émettre de grandes quantités de $\mathrm{CO} 2$ et on rend ainsi nettement plus difficiles et plus coûteuses les velléités futures de réduire les émissions. 
Le débat européen actuel sur la protection du climat pourrait bien remettre en question cette pratique.

Pour finir, qu'il nous soit permis de faire quelques remarques concernant l'importance $\mathrm{du}$ thème de l'environnement dans la géographie économique. Alors que les questions concernant le secteur économique de la protection de l'environnement, la politique énergétique, ou la problématique des ressources sont déjà depuis longtemps abordées dans les disciplines voisines (économie, sociologie de l'industrie, sciences politiques) et ont trouvé place dans les manuels d'enseignement, ce champ d'étude a été longtemps négligé par la géographie économique internationale. Ce n'est que dans les dernières années qu'une série de collègues ont attiré l'attention sur ce thème par des recherches et des publications dans des revues reconnues et des ouvrages collectifs (Angel 2000, Braun et al. 2003, Hayter, Le Heron 2002, Soyez 2002, Gibbs 2006, etc.). Il est alors clair que la géographie économique avec ses outils conceptuels et empiriques donne accès sans problème aux cercles économiques régionaux, au management des flux de matières, aux créations d'entreprises du secteur de la protection de l'environnement jusqu'aux questions de la politique de protection de l'environnement global dans le contexte d'une chaîne de valorisation (Greening of Supply Chains). Une petite partie de ce débat disciplinaire est porté par des collègues allemands. En collaboration avec Dietrich Soyez de l'université de Cologne, l'auteur de cet article a pu avec le soutien d'un groupe de géographes économistes renommés, organiser en mai 2004 la première conférence internationale sur la géographie économique de l'environnement. Celle-ci devait se prolonger en mai 2007 à Storrs aux Etats-Unis (http://www.geography. uconn.edu/ICEEG/), mais cette conférence a été récemment reportée en 2008.

En Allemagne, il existe aussi un colloque de doctorants initié par les universités de Bamberg et de Cologne consacré à la géographie économique orientée vers l'environnement (http://web.uni-bamberg.de/ ba5gi99/Lehrstuhl1/doktoranden/ doktoranden.htm), ainsi qu'un groupe de travail reconnu par la Société Allemande de Géographie sur la recherche en géographie de l'énergie (http://www.uni-tuebingen.de/ egoinfo/akge/).

Ces initiatives sont citées à titre d'exemple car elles témoignent d'un intérêt croissant de la géographie économique pour les questions d'environnement. Cette problématique est de plus en plus reconnue comme étant une possibilité de montrer l'importance politique et dans la vie quotidienne de la recherche géographique, souvent en collaboration avec des groupes de travail de géographie physique (notamment dans les questions des risques environnementaux, de la vulnérabilité, du changement climatique). Le potentiel d'une collaboration fructueuse n'est certainement pas épuisé.

\section{BIBLIOGRAPHIE}

ANGEL D. (2000). - “Environmental Innovation and Regulation”. In : Clark, G.L., Feldman M.P., Gertler, M.S. (éds.), The Oxford Handbook of Economic Geography, Oxford, Oxford University Press, p. 607-622. 
BMU (2007) = Bundesministerium für Umwelt (éd.). - "Entwicklung der erneuerbaren Energien im Jahr 2006 in Deutschland",

http://www.erneuerbare-energien.de/files/pdfs/allgemein/application/pdf/

hintergrund_zahlen2006.pdf 20 avril 2007.

BRAUN B. (2003). - Unternehmen zwischen ökologischen und ökonomischen Zielen. Konzepte, Akteure und Chancen des industriellen Umweltmanagements aus wirtschaftsgeographischer Sicht, Münster. LIT-Verlag (Wirtschaftsgeographie 25), 360 p.

BRAUN B. (2004). - “Der ökologische Umbau der Industrie”. In : Leibniz-Institut für Länderkunde (éd), Nationalatlas Bundesrepublik Deutschland : Unternehmen und Märkte, Heidelberg, Elsevier/ Spektrum Akademischer Verlag, p. 148-149.

BRAUN B. (2005). - "Building global institutions : The diffusion of management standards in the world economy - an institutional perspective". In : Alvstam C.G., scHAMP E.W. (éds), Linking Industries Across the World: Processes of Global Networking, Aldershot, Ashgate, p. 3-27.

BRAUN B., SCHULZ C., SOYEZ D. (2003). — « Konzepte und Leitthemen einer « ökologischen Modernisierung » der Wirtschaftsgeographie ». Zeitschrift für Wirtschafts geographie, 47 (3+4), p. 231-248.

BUND (2006a) = Bund für Umwelt und Naturschutz Deutschland (éd.). - Umwelt und Beschäftigung 2006, Berlin, BUND, 66 p.

BUND (2006b) = Bund für Umwelt und Naturschutz Deutschland (éd.) - Stellungnahme des BUND zum Entwurf des Nationalen Allokationsplans 2008 bis 2012, Berlin, BUND, 9 p., http://www.bmu.de/emissionshandel/downloads/doc/37093.php, 26 octobre 2006.

BWE (2007) = Bundesverband Windenergie e.V. (éd.). - "Datenblatt 2006", http://www. wind-energie.de/fileadmin/dokumente/Hintergrundpapiere/ HG_Datenblatt_2006.pdf, 12 mars 2007

BWE/VDMA (2007) $=$ Bundesverband Windenergie e. V/ Verband Deutscher Maschinen- und Anlagenbau e.V. (éds.). - "Deutsche Wind-Industrie - Export", http://www.deutsche-windindustrie.de/fakten/export/index.html, 12 mars 2007

DESTATIS (2003) = Statistisches Bundesamt Deutschland (éd.). - Energieverbrauch und Luftemissionen des Sektors Verkehr (Kurzfassung), Stuttgart, Metzler-Poeschel, 36 p.

DESTATIS (2005a) = Statistisches Bundesamt Deutschland (éd.). - "Wassergewinnung und Abwassereinleitung 2001", http://www.destatis.de/basis/d/umw/umwtab3.php, 10 octobre 2006

DESTATIS $(2005 b)=$ Statistisches Bundesamt Deutschland (éd.). - "Bodennutzung nach wirtschaftlichen Aktivitäten", http://www.destatis.de/basis/d/umw/ugrtab9.php, 10 octobre 2006

DESTATIS $(2005 \mathrm{c})$ = Statistisches Bundesamt Deutschland (éd.). - "Direkter Energieverbrauch nach Wirtschafts bereichen im Inland", http://www.destatis.de/basis/d/umw/ugrtab3.php, 10 octobre 2006

DESTATIS (2006a) = Statistisches Bundesamt Deutschland (éd.). - "Volkswirtschaftliche Gesamtrechnungen", http://www.destatis.de/basis/d/vgr/vgrtab14.php, 26 août 2006 DESTATIS $(2006 \mathrm{~b})$ = Statistisches Bundesamt Deutschland (éd.). - "Umweltstatistische Erhebungen", http://www.destatis.de/basis/d/umw/umwtab25.php, 26 août 2006 
EC (2006) = European Commission (éd). - "EMAS Statistics and Graphs",

http://ec.europa. eu/environment/emas/pdf/5_5articles_en.pdf, 30 août 2006

GIBBS, D. (2006). - "Prospects for an Environmental Economic Geography: Linking Ecological

Modernization and Regulationist Approaches", Economic Geography, 82 (2), p. 193-215.

HAYTER, R. /LE HERON, R. (2002, éds.). - Knowledge, Industry and Environment. Institutions and innovation in territorial perspective. Aldershot, Ashgate, $420 \mathrm{p}$.

HEYMANN E. (2006). - Umweltsektor China. Von Großbaustelle zum Wachstumsmarkt. Frankfurt am Main, Deutsche Bank Research, 12 p.

Intersolar (2006) = Pressestelle Intersolar (éd.). - "Deutsche Solarwirtschaft auf der Überholspur, Pressemitteilung vom 2.3.2006",

http://www.intersolar.de/61+M5609c4ddad4.html, 15 mars 2007

MOFFET J., BREGHA F. (1999). - "Responsible care". In : Gibson R.B., Voluntary initiatives. The new politics of corporate greening, Peterborough, Broadview Press, p. 69-92.

schulz C. (2005a). - "Foreign Environments : The Internationalisation of Environmental Producer Services", The Service Industries Journal, 25 (3), p. 337-354.

schulz C. (2005b). - Agenten des Wandels ? Unternehmensbezogene Umweltdienstleister im industriellen Produktionssystem. Hochschulschriften zur Nachhaltigkeit 21. München : ökomVerlag, $284 \mathrm{p}$.

SOYEZ D. (2002). - "Environmental Knowledge, the Power of Framing and Industrial Change". In : HAYTER, R. /LE HERON, R. (2002, éds.). - Knowledge, Industry and Environment. Institutions and innovation in territorial perspective. Aldershot, Ashgate, p. 187-208.

SOYEZ D., SCHULZ C. (2002, éds.). - Wirtschaftsgeographie und Umweltproblematik. Köln, Geographisches Institut der Universität zu Köln (= Kölner Geographische Arbeiten 76), 118 p.

TÜV (2005) = TÜV Rheinland Group China (éd.). - "ISO 14001 Environmental management systems", http://www.chn. tuv.com/eng/services/certification/systems/iso14001.html, 22 avril 2005 UBA (2006a) = Umweltbundesamt (éd.). - Wirtschaftsfaktor Umweltschutz. Leistungsfähigkeit der deutschen Klima- und Umweltschutzwirtschaft im internationalen Vergleich, Dessau, Umweltbundesamt, $137 \mathrm{p}$.

UBA (2006b) = Umweltbundesamt (éd.). - "Umweltdaten Deutschland online. Emissionen ausgewählter Luftschadstoffe nach Quellgruppen in Deutschland 1990 bis 2003”, http://www.env-it.de/umweltdaten/public/document/downloadImage.do? ident=7364, 30 août 2006

UBA (2006c) = Umweltbundesamt (éd.). - "Umweltmanagementsysteme weltweit", http://www.umweltbundesamt.de/uba-info-daten/daten/ums-welt.htm, 30 août 2006

Umweltallianz (2006). - “Allgemeine Informationen zu EMAS”, http://www.umweltallianz.de/konkret/emas/allgemein/index.php, 25 mai 2006

Umweltgutachterausschuss (2006a). - “EMAS-Teilnehmerzahlen in den einzelnen Bundesländern", http://www.uga.de/images/stat-bl.jpg, 30 août 2006

Umweltgutachterausschuss (2006b). - "Beteiligung an EMAS in Deutschland nach Branchen", http://www.emas. de/images/uploads/Image/branchen0805.pdf, 30 août 2006 


\section{RÉSUMÉS}

Cet article est consacré à l'étude de l'évolution récente de la protection de l'environnement dans l'industrie manufacturière en Allemagne. En dehors de la situation actuelle de la pollution, on éclaire les mécanismes qui ont contribué à un changement majeur dans les stratégies des entreprises. Une attention particulière est accordée au développement d'un secteur économique de la protection de l'environnement dont les produits et les services sont depuis longtemps devenus des fleurons des exportations allemandes. En conclusion, on propose un regard critique que reflète le récent changement politique.

This article is dealing with the recent development of the environmental performance among Germany's manufacturing industry. Besides the current environmental situation, particular attention is given to the mechanisms that led to a considerable change in corporate strategies. Furthermore, the emergence of an independent environmental industry is highlighted whose products and services have become a success factor of Germany's export activities. The article ends with a critical outlook which also reflects the recent political changes.

Der Beitrag beschäftigt sich mit der jüngeren Entwicklung des industriellen Umweltschutzes in Deutschland. Neben der aktuellen Belastungssituation werden insbesondere diejenigen Mechanismen beleuchtet, die zu einem grundlegenden Wandel in den unternehmerischen Strategien beigetragen haben. Besonderes Augenmerk gilt der Entstehung einer eigenständigen Umweltschutzwirtschaft, deren Produkte und Dienstleistungen längst zu einem Exportschlager geworden sind. Ein kritischer Ausblick, der unter anderem die letzten politischen Wechsel reflektiert, rundet den Beitrag ab.

\section{INDEX}

Keywords : environmental performance of manufacturing firms, corporate environmental policy, incentive and voluntary initiative, environmental industry, exportation of know-how Mots-clés : protection de l'environnement dans l'industrie manufacturière, politique environnementale des entreprises, instrument d'incitation et initiatives volontaires, industrie de l'environnement, exportation de savoir-faire

Schlüsselwörter : Betrieblicher Umweltschutz, industrielle Umweltpolitik, Anreizinstrumente und Selbstverpflichtungen, Umweltschutzwirtschaft, Know-How-Export

\section{AUTEUR}

\section{CHRISTIAN SCHULZ}

Université du Luxembourg- Laboratoire de Recherche en Géographie et Aménagement du Territoire - 162a, avenue de la Faïencerie -1511 Luxembourg- http://www.geo.ipse.uni.lu 